\title{
Totipotent Primordial Germ Cell
}

National Cancer Institute

\section{Source}

National Cancer Institute. Totipotent Primordial Germ Cell. NCI Thesaurus. Code C45734.

One of the earliest cells of an org anism that will be able to reproduce that kind of organism. It is able to develop into any variety of germ cell. 